\title{
Une famille de relatives non standard
}

\author{
Lucien Kupferman \\ Université de Tel-Aviv, Israël \\ kupferma@post.tau.ac.il
}

Résumé. Les relatives extraposées à partir de DP définis (v. Kupferman 2016) et les relatives prédicatives présentent non seulement cette caractéristique remarquable de répondre à un nombre important de réactifs distinctives semblables, mais aussi d'entrer dans un ensemble bien plus inclusif de constructions dotées de propriétés syntaxiques et interprétatives quasiment identiques. Toutes ces relatives non standard décrivent des scènes se déroulant dans une perception non agentive. Les éléments recteurs, verbes ou nominaux, de ces relatives renvoient à des prédicats qui introduisent ces scènes dans la situation. Dans une séquence formée d'un DP tête externe («antécédent») suivi d'une relative non standard, cette dernière fonctionne comme prédicat, et le premier comme son argument dans un système de prédication seconde. Une telle phrase complexe s'analyse ainsi sur deux niveaux : une structure argumentale de prédication seconde, et une structure syntaxique où le prédicat secondaire est projeté sous forme de relative (non standard) et l'argument secondaire sous forme de DP objet du VP primaire. L'analyse présentée veut supplanter, arguments à l'appui, la description traditionnelle $\mathrm{d}$ cas particulier des relatives prédicatives selon laquelle elles seraient dérivées de complétives, le complémenteur étant lui-même converti en qui sujet.

\begin{abstract}
Extraposed relative clauses from definite DPs (see: Kupferman 2016) and predicate relative clauses show up as exhibiting a large array of similar distinctive properties, but, moreover, they enter also into a major set of syntactic and interpretative constructions. All those relative clauses denote scenes which take place under a non agentive perception. Verbal or nominal items governing these relative clauses appear as predicates which function as setting factors of those scenes over the particular situation. Therefore, such a complex-sentence should be analyzed over two levels : an argument structure of secondary predication, and a syntactic structure, where the secondary predicate is projected as a (non standard) relative clause and the secondary argument is projected as an object DP of the primary VP.In a string formed by 1) an external head DP and 2) a non standard relative clause, 2) functions as a predicate, and 1) as its argument in a secondary predication mechanism. The submitted analysis wishes to overcome a more traditional description of the particular case of
\end{abstract}


the predicative relative clauses, according to which they ought to be derived from complement clauses, where the complemetizer is converted into a subject qui.

\section{Sigles utilisés :}

$\mathbf{D P}=$ groupe nominal déterminé $\quad \mathbf{N P}=$ groupe nominal non déterminé $\mathbf{V P}=$ groupe verbal $\mathbf{A P}=$ groupe adjectival $\mathbf{C P}=$ proposition $\mathbf{C}=$ complémenteur $\quad$ Spec, $\mathbf{C P}=$ spécifieur de proposition (=relatifs/ interrogatifs,...).

$\mathbf{R} \mathbf{R}=$ relative restrictive $\quad \mathbf{R A}=$ relative appositive $\quad \mathbf{R P}=$ relative prédicative $\mathbf{R}_{n \boldsymbol{s}}=$ relative non standard $\mathbf{R E D}=$ relative extraposée de DP défini

$\mathbf{S C}=$ petite proposition (=proposition averbale)

$(\mathrm{A}+\mathrm{B})=$ alternance de suites d'éléments de types $\mathrm{A}$ et $\mathrm{B}$ au point saisi dans la séquence $(* \mathrm{~A}+\mathrm{B})=$ dans une séquence, $\mathrm{A}$ est irrecevable, et $\mathrm{B}$ recevable ]

Une littérature linguistique récente décrit (v. par ex. Kupferman 2016) un ensemble particulier de relatives, les relatives extraposées de définis (ci-après : RED). Par leurs propriétés, ces propositions se distinguent des deux ensembles standard de relatives (des adjoints à des nominaux) : les restrictives $(=\mathrm{RR})$ et les appositives $(=$ RA). Cette communication veut montrer que les RED sont en fait un sous-ensemble dans un ensemble de relatives non standard $\left(=R_{n s}\right)$.

En premier lieu, on montre (section 2) que les particularités qui caractérisent les RED les rapprochent fortement des relatives prédicatives (= RP). Les RED et les RP appartiennent à un ensemble plus large encore, qu'on désigne comme relatives non standard $\left(=\mathrm{R}_{n s}\right)$.

En second lieu, la section 3 présente une typologie de ces $\mathrm{R}_{n s}$, et fait apparaître une grande variété de positions dotées de similitudes structurales et interprétatives fortes. On propose pour l'ensemble des $\mathrm{R}_{n s}$ une configuration générale commune représentant une projection syntaxique de séquences à prédication seconde : une inclusion dans des « petites propositions » $(=\mathrm{SC})$.

La section 4 expose alors une réfutation d'une thèse courante dans la littérature, différente du modèle $\mathrm{SC}$, qui veut que les relatives $\mathrm{RP}$ soient des produits dérivés de propositions complétives. On propose en revanche un cadre où les catégories décisives sont une structure argumentale de la prédication seconde et une structure syntaxique où elle est projetée comportant les SC.

Enfin, la section 5 exploite la distribution acquise sur deux niveaux, structure argumentale et structure syntaxique, des constructions à $\mathrm{R}_{n s}$ pour montrer que les interactions entre ces deux niveaux donnent la clé du confinement du relatif tête des $\mathrm{R}_{n s}$ à la seule position de qui sujet. 


\section{Les $\mathbf{R}_{n s}$ : positions et propriétés}

La riche littérature relative aux RP (v. les références suivant ce texte) a monopolisé l'attention sur cet ensemble en le classant comme le troisième type exclusif des propositions relatives, aux côtés des RR et des RA. Cet isolement des RP dans le troisième type a été mis à mal dans Kupferman 2016 qui a traité des RED et a indiqué que leurs propriétés sont très souvent identiques à celles des RP. En fait, RED et RP ne sont que des cas particuliers, des sous-ensembles, dans un ensemble plus vaste: les relatives non standard, ou $\mathrm{R}_{n s}$, dont on va décrire à présent les positions et les propriétés.

Au préalable, on repère les $\mathrm{R}_{n s}$ par un réactif : la propriété qu'ils ont de cataphoriser les séquences DP-relatives, même si les DP dénotent des individus, par le démonstratif non fléchi ça, et non par $(c e l u i+c e l l e+c e u x+c e l l e s)-(c i+l a ̀)$. Cette particularité signale qu'une séquence DP-relative non standard n'est pas un DP complexe - où la relative serait un modifieur (=un adjoint) du DP.

(1) il y a justement (ça+*celle-là), Léa qui danse sur l'estrade hier Ida avait (ça+*celle-là), sa fille qui était malade à la maison ça, Dana qui ne rentrait pas à l'heure, ça l'a mise hors d'elle leur idée pour la scène était (ça+*ceux-là), Marie et Max qui dansent une java

Ida a imaginé (ça+*ceux-là), ses cousines qui jouaient au ping-pong

On est donc bien en présence de relatives qui ne sont pas des adjoints. Leur fonctionnement demande une description distincte.

\subsection{Positions de DP-R $R_{n s}$.}

En plus des positions RP (v. Kleiber, etc.) et RED (v. Kupferman 2016), on observe pour les séquences $\mathrm{DP}-\mathrm{R}_{n s}$ les positions suivantes :

\subsubsection{Positions sous verbes existentiels}

Les séquences $\mathrm{DP}-\mathrm{R}_{n s}$ peuvent être gouvernées par des verbes ou pseudo-verbes existentiels : il y a, avoir, être, voici. Ces verbes matrices renvoient alors à des états transitoires.

(2) a. (il y a+et voici soudain) ces trois chaises qui manquent !

Il y a (ça $+*$ celles-là) : ces trois chaises qui manquent

b. elle a la joue droite qui enfle toujours plus elle a (ça+*celle-là) : la joue droite qui enfle toujours plus

c. la table a son tiroir qui n'ouvre plus la table a (ça+*celui-là) : son tiroir qui n'ouvre plus

d. A : que se passe-t-il ? B : c'est Tom qui demande à vous voir !
A. que se passe-t-il ?
B. c'est (ça+*celui-là) : Tom qui demande à vous voir 


\subsubsection{Positions de sujet}

(3) (ça+*celle-là), Léa qui danse nu-pieds sur l'estrade, ça serait une (* personne+ scène) impressionnante

\subsubsection{Positions régies par des nominaux}

(4) notre ami a été ému par (ça+*celle-là) : cette (*écharpe+vision) de Léa qui dansait nu-pieds sur l'estrade une (*voiture+vidéo) de Paul et de Léa qui s'embrassent fougueusement circule en ce moment

\subsubsection{Positions de prédicat propositionnel}

(5) la meilleure scène a été (ça ${ }^{*}$ celui-là $)$ : Max $(* \emptyset+$ qui implorait Ida)

2.1.5 Positions détachées internes. Une RA - dans (6b) - est admise

(6) a. leur (*voisine+spectacle), Léa qui dansait nu-pieds sur l'estrade, a eu un gros succès

b. leur voisine, qui dansait nu-pieds sur l'estrade, a eu un gros succès

\subsubsection{Positions détachées frontales}

monde

(7) Léa qui dansait nu-pieds sur l'estrade, (*elle+ça) ravissait tout le

\subsubsection{Positions en construction absolue}

(8) avec (ça+celle-là) : Léa qui danse nu-pieds sur l'estrade, Max est ravi

\subsubsection{Positions en phrases simples exclamatives} l'estrade!

(9) Ah quel spectacle que (ça+*celle-là), Ida qui dansait nu-pieds sur

\subsubsection{Pseudo-clivage}

(10) $(*$ celle + ce $)$ que nous imaginions, c'était Léa qui dansait nu-pieds sur l'estrade

hier, $(*$ celle+ce $)$ qu'avait Ida, c'était sa fille qui était malade

$\left({ }^{*}\right.$ celui + ce) qui a ravi notre ami, c'est Dan qui entonnait une tyrolienne

$\left(*^{*}\right.$ celui + ce) qui, comme scène particulièrement tragique, nous a horrifiés, c'est Sam qui se débattait dans un tourbillon ${ }^{\mathrm{i}}$

\subsubsection{Cliticisation du DP objet sans la relative}


(11) sa joue gauche, Eva l'avait qui enflait toujours plus

les voici qui remontent de la cave

La taxinomie exposée ici a permis de délimiter le territoire des $\mathrm{R}_{n s}$, et de montrer que, contrairement aux RR et aux RA, elles ne mettent pas en jeu des configurations d'adjonction (à des DP ou à des NP). La nature de la configuration de DP- $\mathrm{R}_{n s}$ sera débattue à la section 3 .

Propriétés interprétatives déclenchées par les $\mathbf{R}_{\mathrm{ns}}$

Les données de 2.1 présentent cette particularité remarquable des nominaux auxquels sont appliquées les séquences $\mathrm{DP}-\mathrm{R}_{n s}$ que ce sont des termes comme : scène, spectacle, vue (de DP), vidéo, mais jamais des termes d'objets ou d'individus. On revient sur cette généralité dans la section 2.2.2.

On énumère les propriétés interprétatives selon les deux propositions impliquées à chaque fois.

\subsubsection{Dans les enchâssées de la famille $\mathbf{R}_{n s}$}

Comme les RED et les RP, les autres relatives de la famille non standard :

2.2.1.1 Renvoient à des scènes qui se déroulent dans la situation particulière ;

2.2.1.2 Doivent donc avoir des prédicats événementiels ;

2.2.1.3 Et renvoyer aussi à des époques contemporaines de celles des matrices qui les déclenchent ;

(12) a. *PROPRIETE / EVENEMENTS

Sue qui (*connaissait+ tout à coup reconnaissait) Sam a été un intermède inattendu

Dana a sa fille qui est (*malicieuse+malade)

la seule idée de Sam qui (*habite +fait des travaux) au-dessus nous dérange

cette idée, Nini et Ida qui sont (*soeurs+choisies), est intéressante

\section{b. *DISTINCTION D'EPOQUES / COÏNCIDENCE D'EPOQUES}

Léa qui (donne+*donnait) la bonne solution est une perspective réjouissante

Elle frémit au vu de Paul qui (*prendra + prend) le tram en marche La table a son tiroir de droite qui (*ouvrira tout de suite+ouvre maintenant)

\section{résultat contemporain d'un événement antérieur :}

il y a Ida qui a trouvé la solution (Ø+et qui lève le doigt)

je la vois qui a, (visiblement+maintenant), trouvé la réponse

c. *RELATIF DE TETE AUTRE QUE $Q U I / D E$ FORME $Q U I$ 
Paul (*à qui Eva+qui) répondait de travers était une scène mémorable ce sacré spectacle, Léa (*devant qui Max +qui) courait à cloche-pied retenait notre attention

la vision de Sam (*de qui Edna+qui) parle en ce moment est plutôt étrange

\subsubsection{Les matrices déclenchent les scènes dénotées par $R_{\mathrm{ns}}$}

C'est ce travail qu'accomplissent certains éléments figurant dans les matrices des types de 2.1: $\quad$ prédicats perceptuels non agentifs (v. section 4) pour les RP, les prédicats localisants des RED, les $\mathrm{N}$ recteurs perceptuels ou iconiques, les verbes existentiels. La simultanéité des époques de la matrice et de l'enchâssée s'explique aussi de cette façon: le déclenchement de la scène perçue dans $\mathrm{R}_{n s}$ est nécessairement contemporaine de celle de la perception voulue par la matrice. Et la matrice, pour placer l'enchâssée dans la situation, doit asserter ce placement, et ne saurait le nier ou l'interroger :

\section{*CADRES DE SCENE NON ASSERTES / ASSERTES :}

(*y a-t-il +il y a) sa voisine qui le demande ( ?)

Anne qui est arrivée hier (*n'a pas+a) été pour nous un événement déplaisant

Ida (*n'a pas+a) sa joue qui enfle toujours plus

le chat (*n'était pas+était) là qui s'apprêtait à sauter

elle (*ne voit pas+voit) Sam qui traverse entre les voitures ${ }^{\mathrm{ii}}$

\subsection{Une vraie famille}

Les similitudes relevées, tant dans 2.2.1 - entre les configurations que dans 2.2.2 entre les lectures des différents types à relatives non standard, demandent leur prise en compte en termes d'ensemble caractérisé et font appel à un traitement unifié. Tous ces types de relatives entrent eux-mêmes dans un même ensemble, celui des relatives non standard dont ils sont chacun un sous-ensemble.

Il reste à présenter un modèle explicatif des propriétés syntaxiques qui ont été répertoriées dans 2.2.1.

\section{Analyse des $\mathbf{R}_{\mathrm{ns}}$ sous $\mathrm{SC}$}

\subsection{La catégorie $S C:$ de $A P$ à $R_{n s}$.}

Les propriétés argumentales et syntaxiques des $\mathrm{R}_{\mathrm{ns}}$ ont fait apparaître le paradoxe suivant :

- d'une part, le pseudo-clivage de la séquence postverbale des constructions à RP englobe tout à la fois le $\mathrm{DP}$ et $\mathrm{R}_{\mathrm{ns}}$, qui fonctionnent donc comme un seul constituant. (Les configurations 
ne tiennent compte que des éléments pertinents pour le propos présent) :

(14) a. [[CP $[$ Spec, C ce que $]$ Léa avait, c'était $[\mathrm{x}$ [DP sa joue gauche $]\left[_{\mathrm{CP}}\right.$ qui enflait toujours ] plus ]] ]]]]

b. [Eva $\left[\mathrm{VP}\right.$ avait $\left[\mathrm{x}\left[\mathrm{DP}\right.\right.$ sa joue gauche] $\left[_{\mathrm{CP}}\right.$ qui enflait toujours plus

Et il faudra déterminer la nature du constituant (petit) $\mathrm{x}$.

- d'autre part, la cliticisation de la séquence postverbale n'inclut pas la relative, qui reste ainsi extérieure au DP objet :

l'avait ]

(15) a. ${ }^{*}[\mathrm{DP}$ [DP sa joue gauche][CP qui enflait toujours plus]], [CP Eva

b. [sa joue gauche], [CP Eva l'avait [CP qui enflait toujours plus

c. [CP Eva [vP avait [DP sa joue gauche ] [CP qui enflait toujours plus ]]]

Le pseudo-clivage et la cliticisation donnent des résultats apparemment divergents sur la sous- configuration postverbale de (14 b)-(15c) si celle-ci contient une relative non standard. C'est ce paradoxe que la thèse $\mathrm{SC}$ se propose de résoudre. On développe la thèse $\mathrm{SC}$ avancée ici et on l'applique à $\mathrm{DP}-\mathrm{R}_{n s}$, avant de discuter (section 4) d'une description différente courante dans la littérature sur la nature du paradoxe (Radford 1975, Kayne 1977, Guasti 1988, Cecchetto 2011 et des travaux consécutifs).

\subsubsection{Prédication seconde, DP-AP, et SC («petite proposition »)}

Dans Kupferman 2004, chap. 4 et dans des études ultérieures, le concept de prédication seconde est appliqué à des phrases qui incluent des AP appelés par la tradition «attributs d'objet». Par exemple, dans une séquence comme : Léa avait sa joue gauche toute rouge. Ce concept est formalisé par le schéma des «petites propositions», étiquetées $\mathrm{SC}$.

La structure argumentale des constructions à prédication seconde a la forme prototypique (16a). Ce schéma signifie que : le prédicat primaire, $X$, a la capacité de sélectionner une prédication seconde comme argument interne ; cette prédication seconde n'est pas articulée en morphosyntaxe sur $\mathrm{X}$ par une expression d'enchâssement (comme que pour les complétives, à ou de pour certaines infinitives, si pour les interrogatives totales, etc.) ; le prédicat secondaire, ici Z, n'est pas catégorisé comme V.

Cette structure argumentale (16a) (très schématisée) est projetée sur la phrase (16b) et lui permet de générer la structure syntaxique (16c). Ainsi, prédication seconde $=$ DP-SC.

secondaire $\mathrm{Z}$ ]]]

(16) a. [Prédication première $\mathrm{W}$ [Prédicat primaire $\mathrm{X}$ ] [Prédication seconde $\mathrm{Y}$ [Prédicat

b. Léa avait sa joue gauche toute rouge. 
c. [Léa [vP [v avait] [sc [DP sa joue gauche ] [AP toute rouge ]]]]

De cette analyse il ressort une structuration à deux niveaux des constructions à prédication seconde, comme suit :

1. La structure argumentale prévoit que la prédication seconde ( $\mathrm{Y} \mathrm{Z} \mathrm{de}$ (16a) ) est l'argument interne du prédicat primaire (X de (16a)), et cette prédication seconde est elle-même constituée d'un prédicat ( $Z$ de (16a), qui est précédé de son $\operatorname{argument}(\mathrm{Y}$ de (16a)).

2. la configuration syntaxique (16c) prévoit que la prédication seconde de (16a) est projetée sur un constituant, à savoir SC, qui peut être gouverné par un V matrice comme dans (16c) précisément. Ce SC s'énonce comme un DP suivi de la projection du prédicat secondaire, ici un AP.

L'hypothèse de cette double structuration prédit que le pseudo-clivage du constituant postverbal SC inclut tant le DP que l'AP, et que la cliticisation engage seulement le DP. Ce qui est vérifié :

(17) a. [Spec,C ce que] Léa avait, c'était [sc sa joue gauche toute rouge]

b. [DP sa joue gauche, Eva [C $1^{\prime}$ '] avait toute rouge

En conséquence, le DP postverbal a un double statut : il est syntaxiquement régi par le $\mathrm{V}$ matrice, d'où sa cliticisation éventuelle sur ce $\mathrm{V}$, et il est l'argument externe (ou « sujet sélectionnel ») du prédicat secondaire, d'où son pseudo-clivage qui inclut aussi ce prédicat secondaire.

Et voici le tournant de notre affaire : l'AP de (16) peut alterner avec une relative, une $\mathrm{R}_{\mathrm{ns}}$. Il s'ensuit que (petit) x de (14) $=\mathrm{SC}$.

(18) a. Léa avait sa joue gauche qui enflait toujours plus

b. [Léa [vP [v avait] [sc [DP sa joue gauche ][CP qui enflait toujours plus ]]]]

\subsection{Similitudes entre séquences DP-AP et séquences DP- $R_{n s}$}

Cette assimilation des DP- $\mathrm{R}_{n s}$ à la prédication seconde se renforce du fait que les positions particulières des $\mathrm{R}_{n s}$ observées dans 2.1.1-2.1.10 de la section 2 caractérisent aussi les séquences de-AP en prédication seconde. (L'élément de permet de désambiguïser les séquences en les marquant comme des constructions à prédication seconde, en excluant l'occurrence d'expressions à AP adjoints aux nominaux (« épithètes ».)

désolant

(19) ces salles de (*blanches+vides désormais) était un spectacle l'attristait

La (*disparition+vue) de trois verres de vides sur sa table

Des verres de (*noirs+vides), ça vous sape le moral

Elle avait (*posé+vu) trois chaises de bancales dans le couloir

les verres étaient là de complétement (*noirs+vides) 
(*le résultat + le spectacle) qui l'a dérangée était des plates-bandes de piétinées

trois chats étaient entrés dans la cuisine (*complétement blancs+visiblement affamés

avec ses parents de (*curieux+furieux), Léa n'en menait pas large l'aise

sans ses parents de (*curieux+furieux), Léa se sentirait plus à

De plus, on peut aussi le constater sur (19), ces AP dénotent, comme les $R_{n s}$, des états épisodiques, en fait des événements de type 'scène'.

Ces équivalences caractéristiques et cumulées entre AP en prédication seconde et $D P-R_{n s}$ motivent une analyse qui place ces derniers dans le cadre de la prédiction seconde, et les inscrivent dans une configuration SC. SC est un ensemble, relativement homogène par ses propriétés, de constructions dont, à leur tour, les AP considérées ici et les $\mathrm{R}_{\mathrm{ns}}$ sont des sous-ensembles.

On peut réunir ces résultats et énoncer que la configuration d'une construction à $\mathrm{R}_{n s}$ comme (20a) est (20b) :

(20) a. Léa a vu Max et Dana qui dansaient une java langoureuse java langoureuse ]]]]

b. [ Léa a [vp vu [sc [DP Max et Dana] [CP qui dansaient une

\section{Les relatives prédicatives ne sont pas dérivées de complétives.}

Une importante littérature préconise une analyse des RP qui les dérive de complétives (v. Radford 1975, Kayne 1977, ....). Le sujet de la complétive serait élevé au rang d'objet de la matrice, et le complémenteur $(=\mathrm{C}) q_{u} e_{2}$ réduit à une position précédant immédiatement le verbe enchâssé, prendrait la forme qui. Cette analyse conduit ses partisans à dénommer les RP «pseudo-relatives ».

Cette description présente les trois avantages suivants : 1 . elle explique pourquoi la séquence DP-RP répond à des réactifs qui lui confèrent un statut propositionnel ; 2 . elle éclaire le fait que la position postverbale dans la matrice répond à des réactifs qui lui confèrent le statut d'objet matrice ; 3 . elle explique pourquoi le relatif tête de l'enchâssée est confiné à la position sujet et reçoit uniquement la forme qui.

Un certain nombre d'objections conduisent pourtant à rejeter cette thèse. Celle-ci est alors remplacée par l'analyse SC exposée à la section 3 qui précède.

\subsection{La nature des prédicats matrices.}

La thèse, qu'on vient de citer, de la complétive sous-jacente à DP-RP ne prend pas en compte la nécessaire délimitation de l'ensemble des prédicats qui sélectionnent la construction. Elle se contente du constat qu'il s'agit de verbes perceptuels. Mais ceci 
est très insuffisant. Les prédicats qui régissent des séquences DP-RP présentent les propriétés suivantes :

\subsubsection{Ils sont des prédicats non agentifs.}

Certes, les prédicats matrices concernés sont typiquement des verbes perceptuels, mais ce sont aussi des prédicats non agentifs :

(21) apercevoir, entendre, imaginer, sentir, surprendre, trouve, voir

Les constructions passives en se faire et à sujets non humains sont souvent un réactif efficace aux lectures non agentives déclenchées par les membres de l'ensemble (21)

(22) une lueur se fit (*regarder+voir) alors à l'horizon

Un cri sourd se fit (*écouter+entendre) derrière le porche

Le froid se faisait déjà (*ressentir+sentir)

Les cultures se firent (*saisir+surprendre) par une gelée précoce au bout de trois jours, une nouvelle contrée se fit imaginer, déjà annoncée par des mouettes ${ }^{\mathrm{iii}}$

L'ensemble des prédicats ainsi révélé coïncide avec celui des prédicats qui sélectionnent des RP :

(23) la lueur, Ida l'a (*regardée+vue) qui se déplaçait ce cri, Sam l'a (*écouté+entendu) qui se répétait à des intervalles réguliers

Ce froid intense, Sam l'a (*éprouvé+senti ) qui lui entrait dans la chair On les a (*très bien distingués+surpris) qui révisaient leur bac

La thèse des complétives sous-jacentes se heurte à cet obstacle.

Ce même ensemble (21) de prédicats sélectionne par ailleurs des arguments qui dénotent, soit des entités, soit des contenus propositionnels, ces derniers pouvant revêtir la forme de complétives - à condition de figurer sous des prédicats non agentifs. Des prédicats matrices perceptuels agentifs ne peuvent pas sélectionner des complétives. La thèse combattue achoppe devant cette faille.

(24) a. Sam a (regardé+vu) un perroquet / Ida a (*regardé+vu) que Max rentrait tard

Ida a (*écouté+entendu) chez Anne que Max reviendrait plus tôt de Mayotte

va a (*retrouvé+trouvé) que ce filon était prometteur Max a (*conçu+imaginé) que Léa partirait avec lui (mais : OK : je conçois très bien que tu puisses prendre une telle décision)

b. Dan l'a (vue+entendue+imaginée+sentie+surprise+trouvée) qui se préparait déjà à partir 


\subsubsection{Ils sont des prédicats résolument non cognitifs}

Mais une différence, plus nette encore peut-être, apparaît entre complétives et DPRP : les lectures des prédicats de la liste (21) peuvent subir un glissement quand ils sélectionnent des contenus propositionnels sous format de complétives, comme dans (25). Ils reçoivent la propriété 'cognitif'. Cette propriété ne leur est pas attribuée si les contenus propositionnels revêtent la forme de DP-RP :

sortir

(25) Dan a (vu+entendu+imaginé+senti+trouvé) qu'elle se préparait déjà à

Devant des complétives, voir peut se gloser par constater, entendre par apprendre, imaginer par supposer, trouver par estimer, etc. Contrairement aux lectures qui ressortent de ces gloses, l'ensemble de verbes épinglé dans (21) et (24b) contient des prédicats non agentifs et sensoriels (=non cognitifs),

\subsection{La perceptivité ne suffit pas pour la rection de complétives}

Si le champ de l'ensemble des prédicats qui gouvernent des complétives ne coïncide pas exactement avec celui des prédicats matrices qui régissent des constructions DPRP (et il faut leur ajouter - biens sûr - les ensembles épistémiques et de dire, etc., la formulation inverse est encore plus vraie. Ainsi apercevoir ou surprendre :

jardin

(26) a. Ida (*a aperçu qu'ils+les a aperçus qui) tentaient d'entrer dans le ensemble

b. Paul (*a surpris qu'ils+les a surpris qui) révisaient leur bac

\subsection{L'ensemble RP est un cas particulier des $\mathbf{R}_{n s}$}

La thèse de la complétive source de DP-RP ne permet pas de rendre compte du fait que l'ensemble des RP n'est pas un cas isolé, mais, comme l'a montré la section 2, il est en fait un sous-ensemble de l'ensemble des relatives non standard avec lesquelles il partage un nombre important de propretés distinctives.

\subsection{Non prise en compte des particularités interprétatives et syntaxiques des RP}

La thèse des complétives sources interdit de rendre compte des particularités interprétatives des matrices et des enchâssées RP décrites par la section 2. Ces contraintes sont d'une façon générale absentes des complétives, y compris de celles dont le prédicat matrice appartient à la liste (21).

Les quatre objections générales qui ont été émises dans cette section 4 devraient suffire à écarter la thèse des complétives sous-jacentes à des séquences DP-RP et à conduire à une autre description des RP, dans le sens du modèle SC.

(Par ailleurs, le temps manque pour une discussion de l'importante contribution de Muller 2011, et du large corpus présenté où les prédicats matrices de RP dépassent le champ de la perceptivité ${ }^{\text {iv }}$ iii.) 


\section{L'exclusivité de qui sujet dans $\mathbf{R}_{n s}$}

La thèse de la configuration SC des séquences DP-Rns permet aussi de donner une explication de l'occurrence exclusive du relatif qui sujet en tête des $\mathrm{R}_{n s}$. On illustre le système de $\mathrm{R}_{n s}$ par le sous-ensemble des RP, ici l'exemple (18a). Sa configuration syntaxique est schématisée dans (18b), et (27c) en représente la structure argumentale :

(27) $a .=(18 a)$ Léa avait sa joue gauche qui enflait toujours plus $\mathrm{b} .=(18 \mathrm{~b})$ [Léa [VP [v avait] [SC [DP sa joue gauche][CP qui enflait toujours plus ]]]]

c. [ [prédication première Léa [prédicat premier avait [prédication seconde sa joue gauche [prédicat secondaire qui enflait toujours plus ]]]]

On rappelle (v. section 3.2) qu'un DP postverbal dans une matrice de RP, ici sa joue gauche, a une double analyse : objet structural (=syntaxique) du V matrice, ici avoir, et argument externe (= « sujet sélectionnel») appliqué au prédicat secondaire, ici enflait toujours plus. Ainsi donc, la projection syntaxique du système de prédication seconde oblige le prédicat secondaire, inclus dans l'enchâssée de SC, à accueillir un sujet - syntaxique donc - qui représente son argument externe : le relatif qui sujet. Le « sujet sélectionnel » - l'argument externe du prédicat secondaire - ne peut donc avoir que la forme de qui sujet.

\section{Conclusion}

Cette étude a abouti aux résultats suivants :

6.1 Les relatives prédicatives $(=\mathrm{RP})$ et les relatives extraposées de définis (=RED) appartiennent à un ensemble, qu'il a été convenu d'intituler : ensemble, ou famille, de relatives non standard $\left(\mathrm{R}_{n s}\right)$. Ces deux sous-ensembles partagent un nombre important de propriétés substantielles qui, ensemble, les distinguent des relatives restrictives ou des appositives

6.2 Les séquences $\mathrm{DP}-\mathrm{R}_{n s}$ se retrouvent dans des positions phrastiques variées et $\mathrm{y}$ développent les mêmes propriétés, syntaxiques et interprétatives. DP- $\mathrm{R}_{n s}$ dénote une scène qui se déroule dans une perception non agentive, et l'élément matrice recteur de la séquence $\mathrm{DP}-\mathrm{R}_{n s}$ renvoie à un prédicat (verbal, nominal) qui introduit cette scène dans la situation.

6.3 DP-R ${ }_{n s}$ sont, le second un prédicat, le premier son argument, d'une prédication seconde, et sont ainsi comptables d'une relation qui met en jeu la structure argumentale de la phrase. Cette prédication seconde est projetée dans la syntaxe sous le format d'une «petite proposition », ou SC. Le DP qui précède la relative satisfait, au niveau syntaxique, le rôle d'objet (face au verbe matrice), et au niveau de la structure argumentale le rôle d'argument sélectionné par le prédicat secondaire. 
6.4 L'analyse devenue traditionnelle des RP comme dérivées de complétives où le complémenteur serait converti en qui souffre de faiblesses cruciales, qui n'ont été que partiellement recensées ici.

6.5 L'obligation imposée au relatif tête des $\mathrm{R}_{n s}$, qui veut que seul qui sujet figure dans cette position s'explique par la combinaison de : 1) la structure argumentale de la prédication seconde, qui assigne au DP qui est postérieur au prédicat primaire le rôle d'argument externe, ou «sujet sélectionnel», du prédicat secondaire, et 2) de la structure syntaxique, qui fait représenter par le relatif cet argument, qui doit par conséquent avoir le rôle de sujet syntaxique du prédicat secondaire, et être formaté par qui.

\section{Références}

Auwera J. ven der, 1985. «The predicative relatives of French perception verbs», in Bolkensteun A.-M et al. (éds), Predicates and Terms in Functional Grammar, Dordrecht, Foris. 219-234.

Cecchetto C., 2011. Relabeling Heads. A unified account for relativization structures, Linguistic Inquiry 42, 4. 511-560.

Guasti M.-T., 1988. La pseudo-relative et les phénomènes d'accord, Revista de Grammaica Generativa 13. 35-57.

Kayne R.S., 1977. Syntaxe du français. Le cycle transformationnel du français, Paris, Seuil.. 126 et suiv.

Kleiber G., 1988. "Sur les relatives du type je le vois qui arrive», Travaux de linguistique 17. 99-115.

Kupferman L., 2016. ( Le+un) chat était dans la tombe qui regardait Quentin : les déterminants d'arguments matrices face aux relatives extraposées, CMLF 2016.

Muller C., 2011. "Les interprétations sémantique de la prédication seconde intégrée : les relatives prédicatives », Langue française 171. 101-116.

Prebensen H., 1982. "Les propositions relatives dites 'attributives' », Revue romane 17.

98-117.

Radford A., 1975. " Pseudo-relatives and the Unity of Subject-raising », Archivum Linguisticum. 32-64.

Rothenberg M., 1979. «La proposition relative prédicative et attributive. Problème de linguistique française », Bulletin de la Société de linguistique de Paris 74. 351-395.

Schwarze C., 1974. "Les constructions du type Je le vois qui arrive », in Rohrer C. et N. Ruwet (éds.), Actes du colloque franco-allemand de grammaire transformationnelle, Tübingen, Niemeyer. 1, 18-30.

\footnotetext{
'Un relecteur admet des énoncés comme : celle que nous imaginions le plus/le mieux, c'était Léa qui dansait nu-pieds. La relative est ici, pour nous, un appositive. Ainsi, elle peut être omise sans difficulté : (celle+*ce) que nous imaginions le plus/le mieux, c'était Léa. D'autre part, elle ne peut pas être antéposée: (ce+*celle) que nous imaginions le plus/le mieux, c'était, qui dansait nu-pieds, Léa en tenue de gitane.
} 
ii Le même relecteur note à juste titre que les interro-négatives s'adaptent tout-à-fait à la rection de RP. Il cite des exemples comme : n'y a-t-pas sa voisine qui la demande?

Les constructions interro-négatives demandent des confirmations assertives, et, contrairement aux interrogatives, équivalent elles-mêmes à des assertives, elles ont donc des valeurs de vérité :

(i) A : n'y a-t-l pas sa voisine qui la demande?

B : mais si ! Elle l'a demandé !

?? mais non ! Elle ne la demande pas

iii Un relecteur propose aussi le prédicable trouver. Ce prédicable présente une difficulté en ce qu'il fonctionne en binôme avec chercher. Si le premier est un prédicable d'achèvement, selon la quadripartition venzlerienne, c'est-à-dire ponctuel, le second est un prédicable d'accomplissement. L'événement trouver est le point d'aboutissement vers lequel tend l'événement charcherI, qui est par ailleurs agentif. Cette propriété déborde sur trouver. Mais trouver peut se voir attribuer le trait non agentif et il peut régi $\mathrm{r}$ des RP. D'où : sans qu'ils s'y attendent, des paillettes d'or se firent trouver par les prospecteurs dans le cours d'eau, et : on les a trouvés dans la pièce du fond qui jouaient tranquillement.

${ }^{\text {iv }}$ Il faudrait en particulier délimiter les champs des RP et des RR dont les différences sont parfois ténues et difficiles à saisir dans un corpus. La première partie de notre section 4 a assez clairement cerné les PROPRIETES DES prédicats recteurs de RP. 